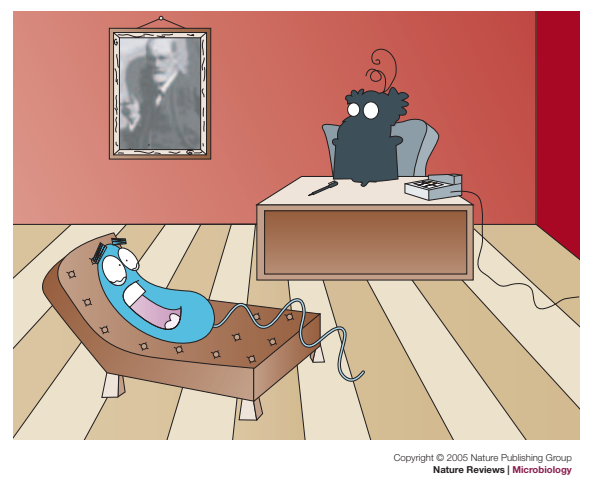

URLS

\title{
Analysing incompatibility - Wolbachia on the couch
}

The obligate intracellular bacterium Wolbachia is renowned for its ability to rapidly invade insect populations using a range of reproductive parasitic mechanisms. One such mechanism is cytoplasmic incompatibility (CI), in which sperm from Wolbachia-infected males is modified so that it can fertilize eggs produced only by females infected with the same Wolbachia strain. As Wolbachia is maternally inherited, the provision of a reproductive advantage to infected females ensures that the bacterium sweeps through insect communities.

Although it has been suggested that CI functions by disrupting karyogamy, little else is known about the molecular basis of this form of reproductive parasitism. Now, a team led by Steven Sinkins in collaboration with Julian Parkhill at the Sanger Institute provides evidence that $\mathrm{CI}$ is facilitated by genes that encode a protein motif, known as an ankyrin (ANK) domain. Importantly, these genes reside on active prophage particles in the Wolbachia genome, a finding that highlights the role of mobile DNA and of horizontal gene transfer in the successful reproductive manipulations of this endosymbiont.

As a starting point in their exploration of CI, Sinkins and coworkers noted that an analysis of the genome of Wolbachia pipientis wPip strain from Culex quinquefasciatus mosquitoes revealed an abundance of genes that encode proteins with
ANK repeats. This motif is unusual in prokaryotes, and the ANK repeat proteins have been implicated in the regulation of the host cell cycle and in cell division. The ANK genes were therefore prime candidates for the mediation of CI. A comparison of the sequence of ANK genes from Wolbachia of the incompatible $C$. quinquefasciatus Bei and Pel strains identified two loci, known as $p k 1$ and $p k 2$, with significant inter-strain DNA and amino-acid-sequence divergence. Variation in the sequence of $p k 1$ and $p k 2$ was also found among other strains of Culex mosquitoes, and one variant of the $p k 2$ gene was expressed in a host-sex-specific manner, providing further evidence of a role for $p k 2$ in CI.

But can adaptation of the insect host affect the outcome of CI? Theoretical models predict that mutations in the host nuclear genome that restore compatibility would be naturally selected and would break down the obstructions to gene flow imposed by CI and, in this study, the authors provide support for this notion. They generated a strain of $C$. quinquefasciatus $\mathrm{P}(w \mathrm{~B})$, which has a Pel nuclear genome, but which harbours Bei Wolbachia. When $\mathrm{P}(w \mathrm{~B})$ females were crossed with Pel males, full compatibility was restored.

Perhaps the most intriguing finding in this report was that both $p k 1$ and $p k 2$ are located in prophage regions of the Wolbachia genome. Furthermore, Sinkins et al. showed that these regions were active
- prophages that contained the variable ANK genes were produced and packaged into phage particles in the host cell.

The finding of putative CI genes on mobile genetic elements has farreaching implications. Many insects that spread human diseases harbour Wolbachia, and scientists have long dreamed of using the bacterium to drive transgenes that inhibit the transfer of disease-causing pathogens through these populations. Unfortunately, the genetic manipulation of Wolbachia has so far proved unsuccessful. Now, however, the authors propose that "Understanding the molecular basis of CI will greatly facilitate its manipulation..." and that these "two variable prophage-associated ANK genes provide valuable markers to aid this process..."

Shannon Amoils

(a) References and links ORIGINAL RESEARCH PAPER Sinkins, S. P. et al. Wolbachia variability and host effects on crossing type in Culex mosquitoes. Nature $\mathbf{4 3 6}$, 257-260 (2005)

FURTHER READING Hoffmann, A. A. Incompatible mosquitoes. Nature 436, 189 (2005) 